\title{
Uma Abordagem para a Modelagem Colaborativa de Processos de Software em Micro e Pequenas Empresas
}

\author{
Marcello Thiry ${ }^{1}$, Christiane Gresse von Wangenheim ${ }^{1}$, \\ Alesandra Zoucas ${ }^{1}$, Kênia Pickler ${ }^{1}$ \\ ${ }^{1}$ Laboratório de Qualidade e Produtividade de Software (LQPS) \\ Universidade do Vale do Itajaí (UNIVALI) - Campus São José \\ Rod. SC 407 Km 4 - 88122-000 - São José - SC - Brasil \\ \{thiry, gresse, azoucas, kenia\}@univali.br
}

\begin{abstract}
This paper describes an approach for the modeling of the software process adapted to small software companies. This work proposes a collaborative model that through workshops promotes the discussion between stakeholders for the modeling and evaluation of the processes. For an initial evaluation, the approach has been applied in 5 companies in Florianópolis/SC.

Resumo. Este artigo descreve uma abordagem para a modelagem de processos de software adaptada para micro e pequenas empresas de software (MPEs). O trabalho propõe um modelo colaborativo que por meio de oficinas promove a discussão entre os envolvidos para a modelagem e validação dos processos. Para a validação inicial da abordagem, ela foi aplicada em 5 empresas em Florianópolis/SC.
\end{abstract}

\section{Introdução}

O setor micro e pequeno empresarial é muito importante para a economia Brasileira atual. Isto tendo em vista que, por exemplo, no setor de software estas empresas representam aproximadamente $70 \%$ do total de empresas e empregam um grande número de pessoas [MCT 2005]. Tipicamente, este tipo de empresa sofre problemas similares a qualquer tipo de empresa, p.ex., no que se refere à qualidade de seus produtos. Porém, no geral, micro e pequenas empresas (MPEs) normalmente enfrentam estes problemas ao extremo devido à informalidade de seus processos e à falta de recursos. Estas características podem prejudicar as MPEs no que se refere à sua qualidade, produtividade e competitividade, ou até mesmo à sua sobrevivência no mercado. Em particular, MPEs têm geralmente um processo de software informal e, conseqüentemente, dependente principalmente da competência das pessoas envolvidas [MCT 2005]. Nesse contexto, o estabelecimento sistemático de processos pode contribuir significativamente na sua melhoria e assim, aumentar sua competitividade e suas chances de sobrevivência. Para estabelecer um processo, o mesmo precisa ser definido e implantado. Ao definirmos um processo é preciso que seja construído um modelo que o represente. Essa representação suporta o entendimento e a visualização do processo, facilita sua disseminação e comunicação, auxilia na gerência de projetos, e é importante na avaliação, evolução e melhoria contínua do processo. 
Para a modelagem de processos, existem várias abordagens [Acuña et al, 2000]. No entanto, a maioria delas está focada em representações formais, voltadas para execução automática do processo, ou para empresas de grande porte. Refinando as abordagens existentes e enfocando especificamente nas características de MPEs (comunicação simples, processos instáveis, necessidade de agilidade, inexperiência na área de engenharia de software, recursos limitados, etc.), nós começamos a desenvolver uma abordagem para a definição de processos de software como parte da abordagem ASPE/MSC [Weber et al. 2005] para o estabelecimento de processos de software em MPEs.

Dentro deste contexto, este artigo apresenta uma abordagem desenvolvida para a modelagem colaborativa dos processos de software de micro e pequenas empresas de software (MPEs). Para a validação inicial da abordagem, ela foi aplicada em 5 empresas da Grande Florianópolis, onde foram modelados os processos de Planejamento de Projeto, Monitoração e Controle de Projeto, e Processo Técnico. Embora acreditemos que a abordagem apresentada seja também adequada para a modelagem de novos processos, ela foi aplicada somente para modelar processos já existentes nas organizações e é dentro deste contexto que os resultados são aqui discutidos.

\section{Abordagem para Modelagem Colaborativa de Processos}

Para poder prover melhoria no processo de software de uma organização, os mesmos devem estar bem caracterizados e compreendidos. Para isso, um conjunto de processos padrão deve ser definido e mantido em cada unidade da organização, juntamente com a construção de guias descrevendo sua aplicabilidade e orientando como customizá-los. Para isto, o processo padrão pode ser documentado explicitamente por meio de um modelo de processo de software que é uma representação abstrata da arquitetura, projeto ou definição do processo de software e que descreve, em diferentes níveis de detalhes, uma organização dos elementos de um processo [Acuña et al, 2000]. Estes modelos de processo podem ser representados com diferentes graus de formalidade e, mais recentemente, em guias de processo voltados para suportar diretamente os executores humanos, os quais se mostraram mais benéficos do que as representações formais pouco usadas na prática [Christie et al, 1997] e [Dingsøyr et al, 2005].

A modelagem de processos pode ser executada de três formas: descritiva, prescritiva ou mista. A modelagem descritiva busca descrever como o processo é executado num contexto particular [Becker et al, 1997] identificando a forma como as atividades são executadas dentro da organização. Por outro lado, a modelagem prescritiva busca descrever como o processo deveria ser executado [Becker et al, 1997], enfocando a melhoria do processo existente com base em boas práticas, modelos de referência e normas da qualidade como, por exemplo, a ISO/IEC 12207 ou CMMI. Outros tipos de modelagem (p.ex., o ASPE/MSC) buscam integrar descrição de processos com algumas prescrições que se fazem necessárias. Porém, este processo de modelagem de processo, especificamente $\mathrm{o}$ levantamento de processo atual dentro de uma empresa caracterizada por processos mais informais e imaturos, se demonstrou complicado na prática requerendo muito tempo e recursos. 
Neste contexto, começamos desenvolver uma abordagem de modelagem colaborativa, que visa a modelagem dos processos de forma incremental a partir de reuniões com a participação de vários envolvidos representando diferentes papéis nestes processos. A abordagem é baseada inicialmente em uma simplificação da técnica Joint Application Design (JAD) [Wood e Silver, 1995] para a identificação de requisitos por meio de seminários altamente focados e nas abordagens práticas para modelagem de processo propostas por [Ahonen et al, 2002] e [Dingsøyr e Moe, 2004].

A abordagem está integrada na abordagem ASPE/MSC, representando um passo integrado em um programa de melhoria. Em passos anteriores, já foram identificados os objetivos de melhoria e, baseados em uma avaliação de contextualização (overview assessment), também foram selecionados os processos a serem definidos. Desta forma, os objetivos da definição dos processos são o levantamento e documentação explícita dos processos de forma descritiva representando como os processos são atualmente executados dentro da unidade da organização. Isto requer a definição de elementos fundamentais dos processos atuais e como eles se relacionam, enfatizando a realidade da aplicação destes processos e evitando que o levantamento seja orientado apenas por políticas da organização ou procedimentos escritos (que talvez não estão sendo seguidos na prática). Além disto, os processos precisam ser documentados de forma detalhada provendo um guia de processo para todos os envolvidos. Um objetivo importante também é a captura ampla dos processos atualmente seguidos (como isto dentro de uma organização mais imatura tipicamente varia de uma pessoa para outra) e a unificação ou representação explícita destas variações (caso haja realmente processos diferentes). Isto também é importante para obter a motivação e comprometimento dos envolvidos como pré-condição para a implementação dos processos num próximo passo.

As principais atividades da abordagem para definição de processo (como parte da abordagem ASPE/MSC):

- Planejamento da modelagem do processo

- Levantamento do processo alto-nível através de oficinas

- Detalhamento do processo

- Revisão do modelo do processo

Planejamento da modelagem do processo. No início, a modelagem do processo é planejada incluindo a seleção do(s) processo(s) a serem modelados, o cronograma, os participantes, a infra-estrutura, etc. Com base neste plano, a modelagem é executada para cada um dos processos a serem modelados.

Levantamento do processo alto-nível através de oficinas. $O$ primeiro passo é o levantamento do processo alto-nível. Seguindo a abordagem colaborativa, este levantamento é feito por meio de oficina(s) junto com representantes de envolvidos no processo e a equipe de melhoria de processo. No início, é feito uma apresentação do objetivo da oficina e da visão geral do processo adotado pela empresa como um todo, obtida a partir de uma avaliação de contextualização (overview assessment) num passo anterior da abordagem ASPE/MSC [Weber et al, 2005] utilizando o método MARES [Gresse von Wangenheim et 
al, 2005]. Em seguida, a técnica de modelagem é brevemente apresentada, incluindo os elementos de notação adotados, os quais são parcialmente baseados na notação UML (vide Tabela 1). 
Tabela 1. Elementos de notação utilizados na abordagem

\begin{tabular}{|c|c|}
\hline & Início de processo \\
\hline & Fim de processo \\
\hline & $\begin{array}{l}\text { Ator responsável: representa o papel responsável pela execução da } \\
\text { atividade }\end{array}$ \\
\hline 0 & $\begin{array}{l}\text { Ator participante: representa os demais papéis que participam na } \\
\text { execução da atividade }\end{array}$ \\
\hline & $\begin{array}{l}\text { Ferramenta ou recurso físico: recursos utilizados para a execução de } \\
\text { uma atividade, p.ex. ferramentas CASE, sistemas de apoio, } \\
\text { ambientes de desenvolvimento, etc. }\end{array}$ \\
\hline & $\begin{array}{l}\text { Atividade: especifica uma seqüência de ações que representa um } \\
\text { comportamento dentro do processo. }\end{array}$ \\
\hline & $\begin{array}{l}\text { Decisão: representa possíveis desvios na seqüência de execução das } \\
\text { atividades. }\end{array}$ \\
\hline & $\begin{array}{l}\text { Artefato: representa todos os (sub-)produtos que são gerados por } \\
\text { (saídas) ou que são consumidos (entradas) por uma atividade. }\end{array}$ \\
\hline & $\begin{array}{l}\text { Fluxo: permite representar a ligação entre as atividades, mostrando a } \\
\text { sequiência de execução. }\end{array}$ \\
\hline & Estado: permite a representação do estado de uma atividade. \\
\hline
\end{tabular}

A partir disto, é feito o levantamento do processo atual com os representantes dos envolvidos no processo. Isto se inicia através da identificação do fluxo de atividades do processo. Neste ponto, a equipe de melhoria atua como facilitador para promover a discussão entre os participantes. De acordo com as informações obtidas pela discussão, é elaborado de forma interativa e incremental um diagrama (vide Figura 1). Para deixar este processo flexível e ágil pode ser usado, por exemplo, um quadro branco magnetizado. A representação dos elementos de notação foi feita em cartolina colorida com o objetivo de facilitar a visualização. Os fluxos dos diagramas foram desenhados no próprio quadro. 


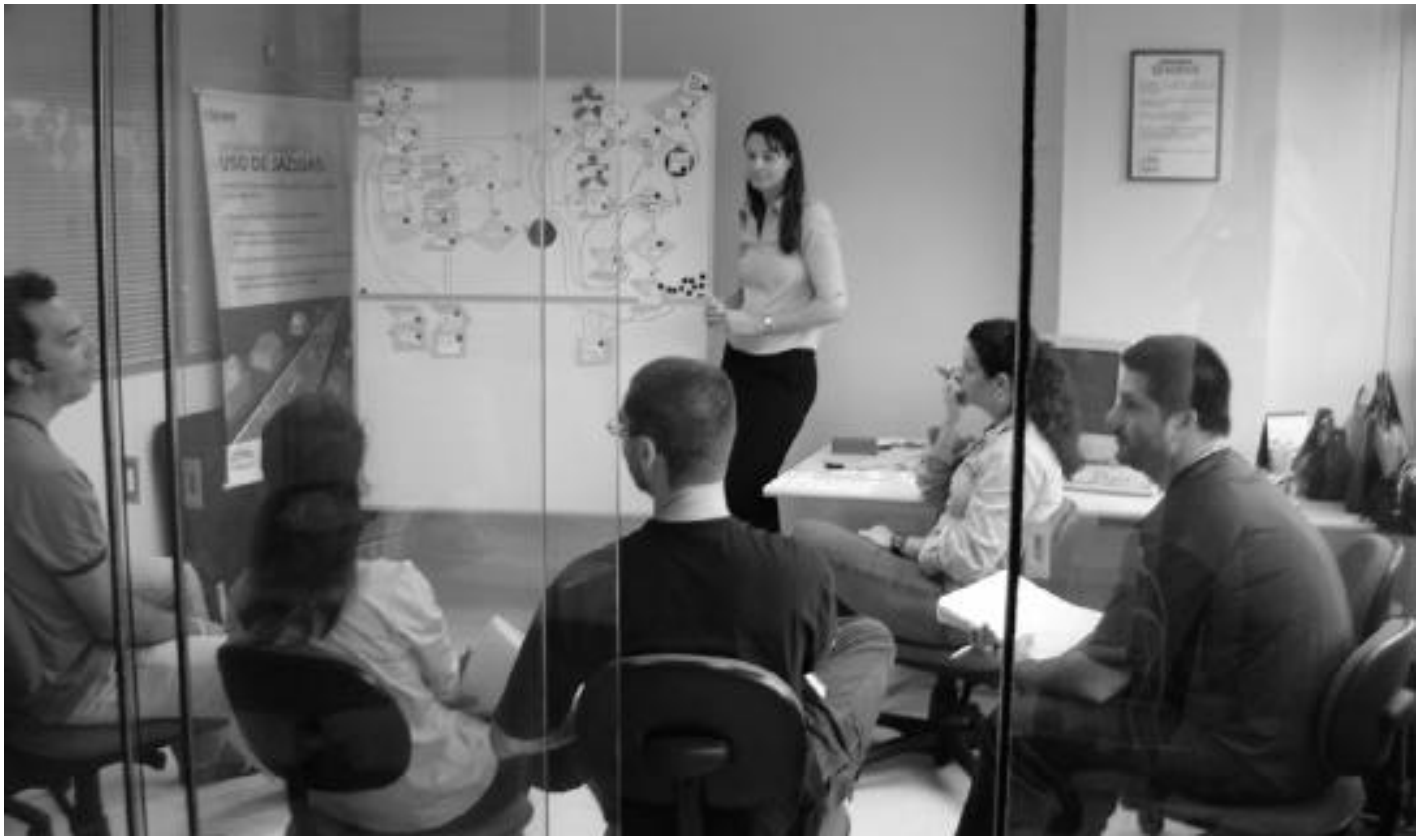

Figura 1. Oficina de levantamento de processo.

A evolução deste diagrama pode ser registrada por meio de fotos digitais (vide Figura 2). Em paralelo, a equipe de melhoria pode também documentar observações adicionais feitas pelos participantes ao longo da oficina.

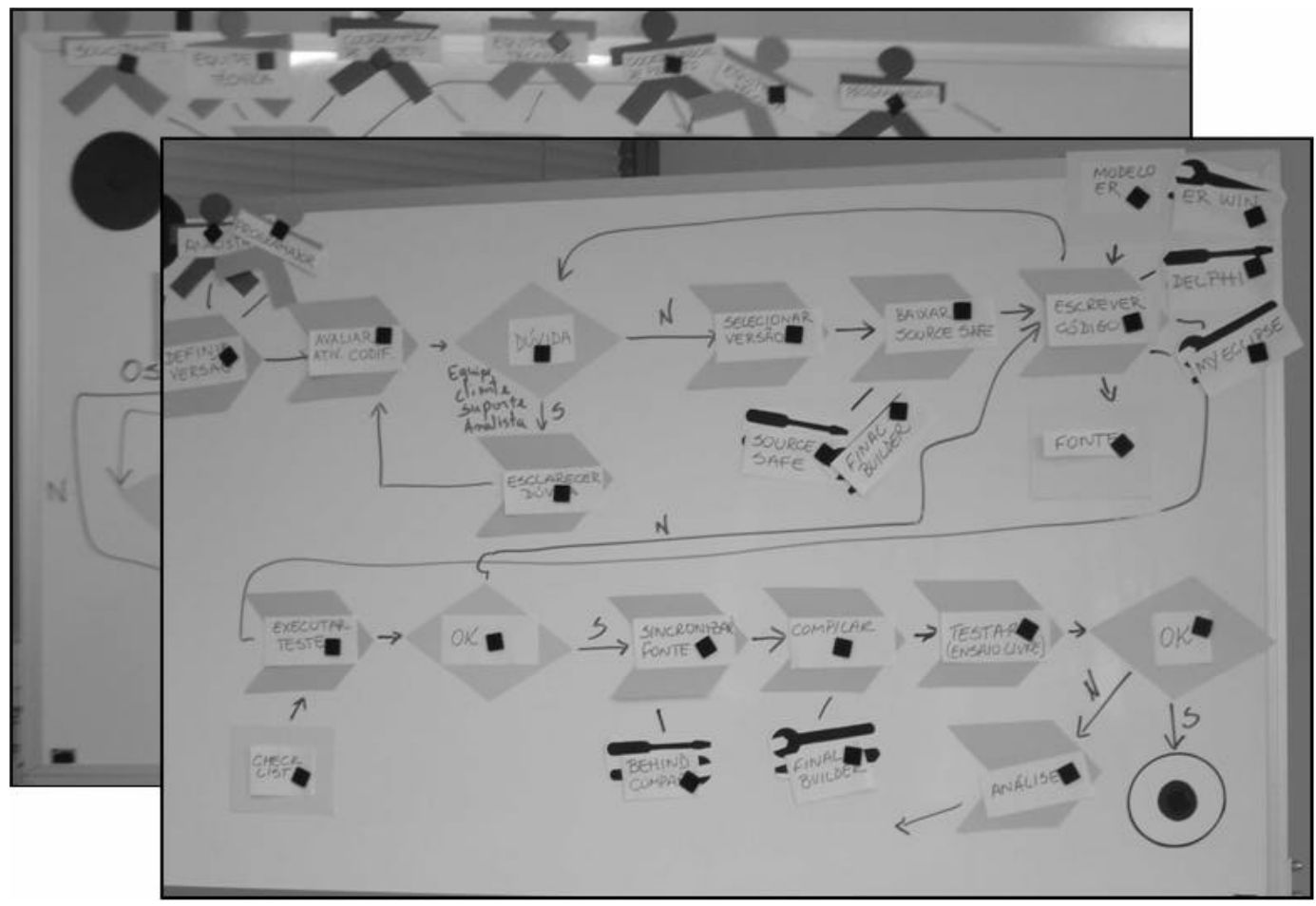

Figura 2. Exemplos do modelo alto-nível no final da oficina. 
A partir das informações levantadas durante a oficina, uma visão alto-nível do processo é documentado (usando, por exemplo, a ferramenta CASE Enterprise Architect) pela equipe de melhoria (vide Figura 3). Dúvidas que sugiram durante a documentação deste diagrama são registradas para uma posterior discussão com os envolvidos durante a oficina de validação.

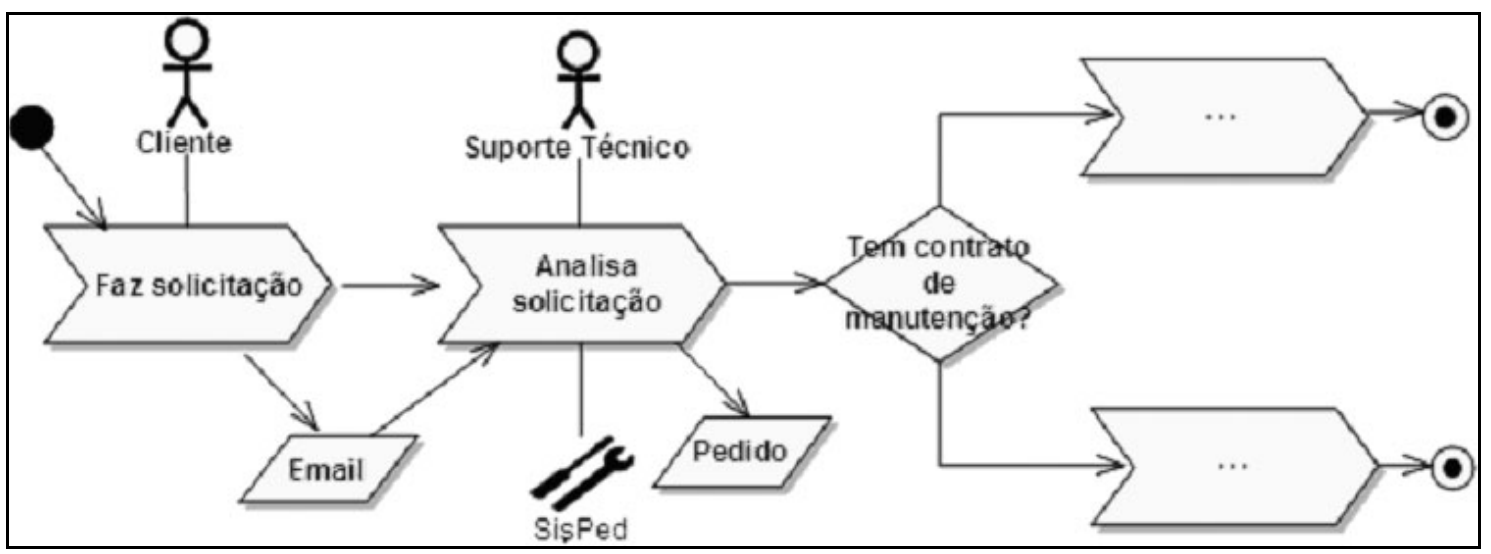

Figura 3. Trecho da modelagem de um processo na ferramenta CASE.

Esta representação alto-nível do processo é validada com representantes dos envolvidos no processo, por meio de uma oficina de validação. O objetivo desta oficina é apresentar e validar a representação do processo alto-nível juntamente com os envolvidos. Com base nas nossas experiências, esta oficina também possibilita uma apresentação/validação, de forma rápida, com mais envolvidos (que não necessariamente participaram da oficina de levantamento), já que a duração desta oficina é mais curta do que da oficina de levantamento. Esta oficina também inicia o processo do detalhamento do processo, apresentando a técnica para o detalhamento de cada atividade a partir da representação alto-nível. Este detalhamento será feito, principalmente, pelos responsáveis de cada uma das atividades.

Detalhamento do processo. A partir da representação alto-nível do processo, cada atividade é detalhada utilizando um template (vide Tabela 2). Este detalhamento é feito pelo(s) responsáveis de uma atividade sendo acompanhada pela equipe de melhoria. A equipe de melhoria também revisa a qualidade do detalhamento do processo em relação da técnica que está sendo utilizada. 
Tabela 2. Template utilizado para o detalhamento das atividades.

\begin{tabular}{|c|c|}
\hline ID & $\begin{array}{l}\text { Para todos os processos deve ser definido um identificador único formado por } \\
\text { letras e números de acordo com o padrão existente na empresa. }\end{array}$ \\
\hline Processo/Atividade & Identificado através do nome do processo usado no diagrama de modelagem. \\
\hline Propósito & Descreve de forma concisa o propósito a ser atingido por este processo. \\
\hline Critérios & Descrevem quando um processo pode começar e terminar. \\
\hline Critério de entrada & Define quando a execução deste processo pode iniciar. \\
\hline Critério de saída & Define quando a execução deste processo pode ser terminada. \\
\hline $\begin{array}{l}\text { Produtos de } \\
\text { trabalho/artefato: }\end{array}$ & $\begin{array}{l}\text { Identificam os produtos de trabalho ou artefatos utilizados e/ou gerados } \\
\text { durante a execução deste processo. }\end{array}$ \\
\hline Entrada(s) & $\begin{array}{l}\text { Identifica qualquer produto de trabalho/artefato utilizado como entrada para } \\
\text { este processo com base nos produtos já identificados no diagrama de } \\
\text { modelagem. }\end{array}$ \\
\hline Saída(s) & $\begin{array}{l}\text { Identifica qualquer produto de trabalho/artefato a ser gerado como saída deste } \\
\text { processo com base nos produtos já identificados no diagrama de modelagem } \\
\text { de processo. }\end{array}$ \\
\hline $\begin{array}{l}\text { Métodos, técnicas e } \\
\text { ferramentas }\end{array}$ & $\begin{array}{l}\text { Identifica qualquer método/técnica (como por exemplo, calculo de pontos de } \\
\text { função, uso da notação UML) e/ou qualquer ferramenta de software em uso } \\
\text { neste processo. }\end{array}$ \\
\hline Responsável & Identifica o papel responsável para a execução deste processo. \\
\hline Papéis envolvidos & Identificam as responsabilidades dos participantes deste processo. \\
\hline Medidas para gerência & $\begin{array}{l}\text { Identifica medidas/dados que estão sendo coletados para sustentar a monitoria } \\
\text { e controle gerencial da execução deste processo. }\end{array}$ \\
\hline Descrição e orientações & Descreve os passos deste processo de forma detalhada. \\
\hline $\begin{array}{l}\text { Orientações para } \\
\text { adaptação }\end{array}$ & $\begin{array}{l}\text { Identifica qualquer necessidade de adaptação dos passos descritos } \\
\text { anteriormente para qualquer situação específica. }\end{array}$ \\
\hline Esforço típico & Identifica o esforço tipicamente gasto na execução deste processo. \\
\hline Duração típica & Identifica o esforço tipicamente gasto na execução deste processo. \\
\hline
\end{tabular}

No final, o detalhamento de todas as atividades de processo é integrado no modelo de processo.

Revisão do modelo do processo. A última etapa da abordagem é a validação conjunta do detalhamento das atividades. Para isto, todo o modelo de processo (incluindo a representação alto-nível e o detalhamento) é disponibilizado para todos os envolvidos no processo possibilitando a revisão do modelo por todos os envolvidos.

\section{Experiências na Prática}

Esta abordagem de modelagem colaborativa de processo foi aplicada em 5 empresas de software em Florianópolis em 2005 no contexto do projeto META 1/PLATIC - Plataforma de Tecnologia da Informação e Comunicação de Santa Catarina, visando a implantação de programas de melhoria de processo na com foco no nível 2 do CMMI-SW em estágios [SEI 2006].

Vale ressaltar que a modelagem dos processos existentes em cada uma das empresas foi parte integrante da fase de diagnóstico do projeto META 1/PLATIC, o qual teve como 
objetivo identificar o estado das práticas adotadas pelas empresas em relação aos seus processos de software. Nenhuma das empresas participantes havia tido experiência com programas de melhoria de software e apenas uma delas possuía algum grau de documentação de seus processos (ainda que bastante alto nível).

Como parte da fase de diagnóstico, no início destes programas de melhoria, foi realizada uma avaliação de contextualização (overview assessment) em cada uma das empresas participantes [GRESSE VON WANGENHEIM et al, 2005] e, a partir dos resultados destas avaliações, foram planejadas as ações de melhoria, iniciando com a modelagem do processo de software existente em cada uma das empresas.

A aplicação foi realizada em empresas de diferentes portes e com características distintas (vide Tabela 3).

Tabela 3. Características das empresas participantes.

\begin{tabular}{|c|c|c|c|c|c|}
\hline & Empresa 1 & Empresa 2 & Empresa 3 & Empresa 4 & Empresa 5 \\
\hline Porte & Micro & Pequena & Pequena & Pequena & Grande \\
\hline Fundação & 1997 & 2001 & 1993 & 1991 & 1990 \\
\hline Área de atuação & $\begin{array}{l}\text { Consultoria em } \\
\text { gestão } \\
\text { organizacional e } \\
\text { implantação de } \\
\text { sistemas. }\end{array}$ & $\begin{array}{l}\text { Produtos e } \\
\text { serviços de } \\
\text { segurança e no } \\
\text { ambiente virtual. }\end{array}$ & \begin{tabular}{|l} 
Desenvolviment \\
o de sistemas e \\
equipamentos \\
para gestão de \\
filas.
\end{tabular} & \begin{tabular}{|l|} 
Desenvolviment \\
o de Sistemas \\
para Call Center \\
e Contact \\
Center.
\end{tabular} & $\begin{array}{l}\text { Produtos para } \\
\text { Construção } \\
\text { Civil, Judiciário, } \\
\text { Administração } \\
\text { Pública, etc. }\end{array}$ \\
\hline Sócios & 2 & 3 & 2 & 4 & 3 \\
\hline Funcionários & 5 & 14 & 16 & 36 & 159 \\
\hline $\begin{array}{l}\text { Principais } \\
\text { metas }\end{array}$ & $\begin{array}{l}\text {-Expandir a faixa } \\
\text { no mercado } \\
\text {-Melhorar } \\
\text { produtividade } \\
\text {-Melhorar } \\
\text { confiabilidade }\end{array}$ & $\begin{array}{l}\text {-Melhorar } \\
\text { produtividade. } \\
\text {-Melhorar } \\
\text { estimativas de } \\
\text { prazos } \\
\text {-Reduzir prazos } \\
\text { de } \\
\text { desenvolviment } \\
\text { o } \\
\end{array}$ & \begin{tabular}{|l|}
-Melhorar \\
produtividade \\
-Reduzir prazos \\
de \\
desenvolviment \\
o \\
-Melhorar \\
manutenibilidade
\end{tabular} & \begin{tabular}{|l|}
-Expandir a faixa \\
no mercado \\
-Melhorar \\
produtividade \\
-Certificação \\
CMMI nível 2
\end{tabular} & $\begin{array}{l}\text {-Expandir a faixa } \\
\text { no mercado } \\
\text {-Melhorar } \\
\text { produtividade e } \\
\text { satisfação dos } \\
\text { clientes }\end{array}$ \\
\hline $\begin{array}{l}\text { Principais } \\
\text { Clientes }\end{array}$ & $\begin{array}{l}\text { Setor de } \\
\text { construção civil }\end{array}$ & $\begin{array}{l}\text { Setores } \\
\text { financeiros, } \\
\text { comerciais e } \\
\text { governamentais. }\end{array}$ & \begin{tabular}{|l|} 
Setores \\
financeiros, \\
comerciais, \\
educacionais e \\
governamentais.
\end{tabular} & $\begin{array}{l}\text { Empresas de } \\
\text { Call Center e } \\
\text { Contact Center }\end{array}$ & \begin{tabular}{|l|} 
- Judiciário. \\
-Construção \\
Civil \\
-Ministério \\
Público \\
\end{tabular} \\
\hline Certificação & Não possui & Não possui & ISO 9000 & Não possui & ISO 9000 \\
\hline
\end{tabular}

A modelagem dos processos das empresas participantes foi realizada seguindo a abordagem apresentada no capítulo anterior. A modelagem foi conduzida principalmente pelos consultores externos (representados pela equipe do LQPS/UNIVALI) em conjunto com o(s) responsável(is) pela melhoria de processo das empresas. Participaram no processo de modelagem, representantes de todos os envolvidos nos processos que foram modelados 
em todos os níveis/papéis. Nestas aplicações, o número de representantes varia de 9 até 12. Com base nas nossas experiências, dependendo do número de envolvidos no processo pode ser de grande importância que todos participem também nas oficinas, já que a própria discussão nas oficinas gera uma maior compreensão com relação ao processo, e possibilitam a resolução de inconsistências/variações diretamente, além de troca de experiências entre os envolvidos. Caso, este número de envolvidos seja muito grande ( $>8$ pessoas) sugerimos envolver, num primeiro momento na oficina de levantamento, somente alguns representanteschave.

Com base no plano de melhoria nas empresas participantes foram selecionados os processos de "Planejamento de Projeto", "Monitoração e Controle" e o Processo Técnico (incluindo o desenvolvimento e gerência de requisitos, projeto (design), codificação e testes, instalação).

Os dados referentes ao número de participantes e duração das oficinas realizadas para o levantamento e validação da representação alto-nível do modelo de processo nas 5 empresas são resumidos nas Tabelas 4 (empresas 1, 2 e 3) e 5 (empresas 4 e 5).

Tabela 4. Quadro resumido com os dados das aplicações realizadas (empresas 1, 2 e 3).

\begin{tabular}{|c|c|c|c|c|}
\hline Empresa & $\begin{array}{c}\text { Principais } \\
\text { Etapas }\end{array}$ & $\begin{array}{c}\text { Planejamento de } \\
\text { Projeto }\end{array}$ & $\begin{array}{c}\text { Monitoração e } \\
\text { Controle }\end{array}$ & Processo Técnico \\
\hline \multirow[t]{2}{*}{1} & Levantamento & \multicolumn{3}{|l|}{$\begin{array}{ll}\sim 2 \mathrm{~h} \\
\text { Participantes } \\
\text { - } \quad \text { Empresa = 3 } \\
\text { - } \quad \text { Avaliadores = 3 }\end{array}$} \\
\hline & Validação & \multicolumn{3}{|c|}{ Não houve necessidade de validação. } \\
\hline \multirow[t]{2}{*}{2} & Levantamento & \multicolumn{2}{|l|}{$\begin{array}{l}\sim 1 \mathrm{~h} \\
\text { Participantes } \\
\text { - } \quad \text { Empresa = 2 } \\
\text { - } \quad \text { Avaliadores = 3 }\end{array}$} & $\begin{array}{l}\text { 2h10m } \\
\text { Participantes } \\
\text { - } \quad \text { Empresa = } 7 \\
\text { - } \quad \text { Avaliadores = 3 }\end{array}$ \\
\hline & Validação & \multicolumn{2}{|l|}{$\begin{array}{l}\sim 2 \mathrm{~h} \\
\text { Participantes } \\
\text { - } \quad \text { Empresa = 4 } \\
\text { - } \quad \text { Avaliadores = 3 }\end{array}$} & $\begin{array}{l}\sim 1 \mathrm{~h} 30 \mathrm{~m} \\
\text { Participantes } \\
\text { - } \quad \text { Empresa = 4 } \\
\text { - } \quad \text { Avaliadores = } 2\end{array}$ \\
\hline \multirow[t]{2}{*}{3} & Levantamento & $\begin{array}{l}\sim 1 \mathrm{~h} 30 \mathrm{~m} \\
\text { Participantes } \\
\text { - } \quad \text { Empresa = } \\
\text { - } \quad \text { Avaliadores = 3 }\end{array}$ & $\begin{array}{l}\sim 0 \mathrm{~h} 15 \mathrm{~m} \\
\text { Participantes } \\
\text { - } \quad \text { Empresa = } 3 \\
\text { - } \quad \text { Avaliadores = } 3\end{array}$ & $\begin{array}{l}\sim 1 \mathrm{~h} 05 \mathrm{~m} \\
\text { Participantes } \\
\text { - } \quad \text { Empresa = } 3 \\
\text { Avaliadores = } 2\end{array}$ \\
\hline & Validação & $\begin{array}{l}\sim 0 \mathrm{~h} 30 \mathrm{~m} \\
\text { Participantes } \\
\text { - } \quad \text { Empresa = 3 } \\
\text { - } \quad \text { Avaliadores = 3 }\end{array}$ & Não foi validado & $\begin{array}{l}\sim 0 \mathrm{~h} 30 \mathrm{~m} \\
\text { Participantes } \\
-\quad \text { Empresa = 4 } \\
\text { - } \quad \text { Avaliadores = 3 }\end{array}$ \\
\hline
\end{tabular}

Pode-se notar que, devido às diferentes características das empresas participantes, a abordagem descrita foi aplicada com algumas pequenas variações. Por exemplo, na empresa 
1, que recentemente criou seu setor de desenvolvimento, os três processos foram levantados em uma única oficina, já incluindo também a validação. Esta flexibilidade da abordagem tornase necessária para garantir a sua adaptação à micro e pequenas empresas. Também é possível observar que a empresa 5 não está classificada dentro deste contexto, tendo um porte maior. Mesmo assim, a abordagem continuou mostrando-se aplicável. Alguns dos processos também não foram ainda levantados/validados em todas as empresas visando a sua modelagem mais integrada já na parte da melhoria dos respectivos processos. A modelagem do processo de Monitoração e Controle da empresa 4 não foi feita de modo explícito, uma vez que ela acabou sendo integrada com a modelagem do processo de Planejamento.

Tabela 5. Quadro resumido com os dados das aplicações realizadas (empresas 4 e 5).

\begin{tabular}{|c|c|c|c|c|}
\hline Empresa & $\begin{array}{c}\text { Principais } \\
\text { Etapas }\end{array}$ & $\begin{array}{c}\text { Planejamento de } \\
\text { Projeto }\end{array}$ & $\begin{array}{l}\text { Monitoração e } \\
\text { Controle }\end{array}$ & Processo Técnico \\
\hline \multirow[t]{2}{*}{4} & Levantamento & $\begin{array}{l}\sim 3 \mathrm{~h} 00 \mathrm{~m} \\
\text { Participantes } \\
\text { - } \quad \text { Empresa = 9 } \\
\text { - } \quad \text { Avaliadores = } 3\end{array}$ & \multirow{2}{*}{$\begin{array}{l}\text { Não foi modelado } \\
\text { explicitamente. }\end{array}$} & $\begin{array}{l}\text { 2h30m } \\
\text { Participantes } \\
\text { - } \quad \text { Empresa = 9 } \\
\text { - } \quad \text { Avaliadores = } 3\end{array}$ \\
\hline & Validação & $\begin{array}{l}\sim 2 \mathrm{~h} 00 \mathrm{~m} \\
\text { Participantes } \\
\text { - } \quad \text { Empresa }=4 \\
\text { - } \quad \text { Avaliadores = } 2\end{array}$ & & $\begin{array}{l}1 \mathrm{~h} 30 \mathrm{~m} \\
\text { Participantes } \\
\text { - } \quad \text { Empresa }=6 \\
\quad \text { Avaliadores }=2\end{array}$ \\
\hline \multirow[t]{2}{*}{5} & Levantamento & $\begin{array}{l}\sim 3 \mathrm{~h} 20 \mathrm{~m} \\
\text { Participantes } \\
\text { - } \quad \text { Empresa = 5 } \\
\text { - } \\
\text { Avaliadores = 4 }\end{array}$ & $\begin{array}{l}\sim 1 \mathrm{~h} 10 \mathrm{~m} \\
\text { Participantes } \\
\text { - } \quad \text { Empresa }=5 \\
\text { - } \quad \text { Avaliadores = } 4\end{array}$ & $\begin{array}{l}\text { Codificação } \sim 1 \mathrm{~h} 30 \mathrm{~m} \\
\text { Teste } \sim 1 \mathrm{~h} 25 \mathrm{~m} \\
\text { Participantes } \\
-\quad \text { Empresa }=6 \\
\text { - } \quad \text { Avaliadores }=3\end{array}$ \\
\hline & Validação & $\begin{array}{l}\sim 1 \mathrm{~h} 55 \mathrm{~m} \\
\text { Participantes } \\
\text { - } \quad \text { Empresa }=5 \\
\text { - } \quad \text { Avaliadores = 4 }\end{array}$ & $\begin{array}{l}\sim 1 \mathrm{~h} 00 \mathrm{~m} \\
\text { Participantes } \\
\text { - } \quad \text { Empresa = } 1 \\
\text { - } \quad \text { Avaliadores = 4 }\end{array}$ & $\begin{array}{l}\sim 1 \mathrm{~h} 50 \mathrm{~m} \\
\text { Participantes } \\
\text { - } \quad \text { Empresa }=6 \\
\text { - } \\
\quad \text { Avaliadores }=2\end{array}$ \\
\hline
\end{tabular}

Com base nestas experiências, podemos doservar que a abordagem apresentada permite de forma rápida modelar o processo existente de uma empresa. As principais vantagens que identificamos são:

- O levantamento e a modelagem do processo "real" da empresa.

- A possibilidade de deixar o processo explícito unificando, completando e identificando também variações de processos que tipicamente existem em organizações com processos mais informais e imaturos.

- A troca de experiências e/ou melhorias que está sendo feita individualmente entre os envolvidos de um processo estimulando um efeito de melhoria e aprendizagem direta.

- A participação dos envolvidos no processo de modelagem aumenta a motivação e comprometimento da equipe uma vez que todos têm a oportunidade de definir o seu próprio processo. 
Ainda com base nas nossas experiências em organizações de vários tamanhos, também observamos, que a abordagem é flexível e pode ser facilmente aplicada principalmente em micro e pequenas empresas.

\section{Discussão}

Neste artigo apresentamos uma abordagem colaborativa para a modelagem de processos de software principalmente em micro e pequenas empresas de software. A abordagem complementa a abordagem ASPE/MSC na parte da definição de processo, oferecendo um meio de fácil de levantar e detalhar os processos atuais da empresa.

A abordagem é baseada inicialmente em uma simplificação e transferência da técnica Joint Application Design (JAD) para a identificação de requisitos por meio de seminários altamente focados. Ela também aplica conceitos de outras abordagens práticas para modelagem de processo propostas por [Ahonen et al, 2002] e [Dingsøyr e Moe, 2004]. Neste contexto, a principal diferença é a sua integração numa abordagem mais abrangente cobrindo toda parte de estabelecimento efetivo de processos (ASPE/MSC) e a definição de uma notação e templates para a documentação do processo.

A notação definida é baseada no SPEM (Software Process Engineering Metamodel) [OMG, 2005], que define um conjunto mínimo de elementos de modelagem para descrever qualquer processo de desenvolvimento de software, bem como seus componentes. Ele busca suprir a necessidade de um padrão para as técnicas de modelagem de processo, utilizando uma abordagem orientada a objetos e UML como notação. Embora a especificação do SPEM seja bastante completa e permita grande detalhamento dos processos envolvidos, considerou-se que sua utilização na prática, envolvendo diretamente os participantes da empresa, com diferentes graus de conhecimento em Engenharia de Software poderia ser um fato inibidor e os objetivos não seriam alcançados. Assim, foi especificada uma notação especificamente voltada para a modelagem de processo de software neste contexto, incluindo também elementos adicionais (como por exemplo, aquele que representa $o$ estado de uma atividade). Porém, cabe ressaltar que o foco deste trabalho não foi estabelecer uma nova notação. A contribuição está muito mais relacionada com a proposta de um trabalho colaborativo envolvendo diferentes papéis dentro da organização para a documentação dos processos em uso.

As nossas experiências com a aplicação da abordagem em 5 empresas de software da Grande Florianópolis, mostraram uma primeira indicação da aplicabilidade da abordagem principalmente no contexto de micro e pequenas empresas. Como resultado da abordagem, foram criados modelos dos processos atuais das empresas participantes que estão sendo atualmente revisados e utilizados como base inicial para a implementação subseqüentes de ações de melhoria.

Neste sentido, com as aplicações em andamento, as empresas perceberam a utilidade de entender seus processos na prática e as similaridades e discrepâncias existentes entre o entendimento de diferentes pessoas. Mais do que isso, elas também confirmaram que este trabalho já era uma melhoria em si e que outras oportunidades tornaram-se visíveis. 
Também identificamos que seria viável adotarmos a mesma abordagem para a modelagem de novos processos. Entretanto, esta atividade exigiria assegurar um conhecimento prévio básico dos participantes sobre a área da Engenharia de Software que estaria sendo modelada. Neste sentido, a modelagem de novos processos precisaria ser considerada no plano de melhoria da organização com uma atividade prévia de treinamento sobre a área em questão.

É possível concluir que a abordagem desenvolvida, embora ainda precise de mais aplicações para ir se ajustando, já permite uma visão detalhada dos processos existentes na organização, com um grau de detalhamento razoável. Por outro lado, este detalhamento ainda não é suficiente para guiar completamente a sequiência de ações de melhoria. Ele precisará ser utilizado em conjunto com os outros resultados já obtidos no projeto, em particular aqueles das avaliações de contextualização [Gresse von Wangenheim et al, 2005].

\section{Agradecimentos}

Os autores gostariam agradecer todos os representantes de empresas que participaram da aplicação da abordagem na prática. Também gostaríamos de agradecer aos órgãos de fomento CNPq, FINEP e FAPESC que suportam o projeto PLATIC, no qual este trabalho está contextualizado.

\section{Referências}

Acuña, S.T.; Antonio, A. De; Ferré, X.; López, M.; Maté, L. The Software process: modeling, evaluation and improvement. Handbook of Software Engineering and Knowledge Engineering, 2000.

Ahonen, J. J., Forsell, M. E Taskinen S. A Modest but Practical Software Process Modeling Technique for Software Process Improvement. Software Process Improvement and Practice, 2002, vol. 7, p.33-44.

Becker, U.; Hamann, D.; Verlage, M. Descriptive Modelling of Software Processes. IESEReport, Alemanha, Dec.1997.

Christie, A.; Levine, L.; Morris, E.J.; Riddle, B.; Zubrow, D.; Belton, T.; Proctor, L.; Cordelle, D.; Ferotin, J.; Solvay, J.. Software Process Automation: Interviews, Survey and Workshop Results. (CMU/SEI-97-TR-008) Pittsburgh: PA: Software Engineering Inst., Carnegie Mellon Univ., October 1997.

Dingsøyr, T. E Moe, N. B. The Process Workshop: A Tool to Define Electronic Process Guides in Small Software Companies. Proceedings of the Australian Software Engineering Conference, IEEE, 2004.

Dingsøyr, T.; Moe, N.B.; Dyba, T.; Conradi, R. A Workshop-Oriented Approach for Defining Electronic Process Guides. In: JURISTO, N.; ACUÑA, S.T. Software Process Modeling, Kluwer Academic Publishers, 2005.

Gresse Von Wangenheim, C., Pickler, K., Thiry, M., Zoucas, A., E Salviano, C. Aplicando Avaliações de Contextualização em Processos de Software Alinhados ao CMMI-SE/SW. 
In: SIMPROS 2005, Simpósio Internacional de Melhoria de Processo de Software, São Paulo, Brasil, 2005.

ISO/IEC Std. 15504: Information Technology - Process Assessment, Part 1 to Part 5. International Organization for Standardization, 2003-2006.

MCT - Ministério de Ciência e Tecnologia (Brasil). Pesquisa sobre Qualidade e Produtividade no Setor de Software, 2005. http://www.mct.gov.br.

OMG - Object Management Group. Software Process Engineering Metamodel Specification. Version 1.1, 2205.

SEI - Software Engineering Institute. Capability Maturity Model Integration (CMMI) Product Suite, 2006. http://www.sei.cmu.edu/cmmi

SOFTEX. Guia Geral do MPS.BR - Melhoria de Processo do Software Brasileiro (Versão 1.0). Abril, 2005.

Weber, S.; Hauck, J. C. R.; Gresse Von Wangenheim, C. Estabelecendo Processos de Software em Micro e Pequenas Empresas. In: SBQS - Simpósio Brasileiro de Qualidade de Software, Porto Alegre, Brazil, 2005.

Wood, J. E Silver D. Joint Application Development (Paperback). Wiley, 1995. 